\title{
Correlation Between Serum CA-125 Level and CT Scan Findings of Abdominal Tuberculosis Resembling Ovarian Mass
}

\author{
${\text { Narendra Wahyu Junior }{ }^{\mathrm{a}} \text {, Lies Mardiyana }}^{\mathrm{a}}$, Bambang Soeprijanto ${ }^{\mathrm{a}}$, Hari Nugroho ${ }^{\mathrm{b}}$ \\ a narendrajuniordr@gmail.com \\ ${ }^{a}$ Department of Radiology, Faculty of Medicine Airlangga University, Dr. Soetomo General Academic Hospital Surabaya, Indonesia \\ ${ }^{b}$ Department of Obstetrics and Gynecology, Faculty of Medicine Airlangga University, Dr. Soetomo General Academic Hospital \\ Surabaya, Indonesia
}

\begin{abstract}
Background: Female patients with clinical abdominal tumors generally undergo abdominal CT scan and serum CA-125 level examination. Thirty-three percent of patients with pelvic inflammatory disease and abdominal tuberculosis also had elevated blood CA-125 level. CT scan, which is an imaging modality with ionizing radiation, is important for diagnosing ovarian tumors. It is very common to find discrepancy between abdominal tumor's preoperative diagnosis and postoperative diagnosis, which turns out to be abdominal tuberculosis. This study aims to determine the correlation between serum CA125 levels with abdominal CT scan findings in abdominal TB resembling ovarian mass.

Methods: This was a correlative analytic study with a retrospective approach which was conducted at Dr. Soetomo General Hospital, Surabaya. The sample of this study was medical records from all patients with abdominal tuberculosis who had undergone histopathological examination. Serum CA-125 levels, surgery, and histopathological examination data were obtained from medical records and statistical analysis was performed.

Results: The distribution of the lesion extension degree varied, but most were included in moderate degree (59.1\%). In terms of CA-125 level, most patients had significant increase (72.7\%) of their CA-125 level. There was a negative correlation (rho $=-0.096)$ which was not significant $(\mathrm{p}=0.671$ with $\alpha=0.05)$ between serum CA 125 -levels and the extension degree of abdominal tuberculosis lesion resembling ovarian tumors.

Conclusion: There is a negative and insignificant correlation between serum CA-125 level and the degree of abdominal tuberculosis lesion extension resembling ovarian tumor.
\end{abstract}

Published by IJRP.ORG. Selection and/or peer-review under responsibility of International Journal of Research Publications (IJRP.ORG)

Keywords: Abdominal Tuberculosis; CA-125 Level; CT-Scan; Ovarian Tumor

\section{Introduction}

In 2014, TB prevalence in Indonesia was 297 per 100,000 population [1]. Meanwhile, abdominal and pelvic tuberculosis prevalence reached $21.7 \%$ of all tuberculosis cases [2]. Abdominal and urogenital tuberculosis were the second most common sites for extrapulmonary tuberculosis. Urogenital tuberculosis could account for $19 \%$ of gynecological visits in some developing countries [3].

Female patients with clinical abdominal tumors generally undergo abdominal CT scan and serum CA-125 level examination. Apart from ovarian cancer, elevated blood CA-125 level might be caused by pancreatic cancer, colon cancer, lung tumors, and benign conditions such as endometriosis and pregnancy [4]. In another study, $33 \%$ of patients with pelvic inflammatory disease and abdominal tuberculosis also had elevated blood CA-125 level [5,6]. In ovarian tuberculosis cases, an increase in CA-125 level could reach above 500 U/ml and a decrease in CA-125 level correlates with the disease resolution by antituberculosis therapy [6]. 
Imaging plays an important role in diagnosing and staging ovarian tumors [7]. CT scan, which is an imaging modality with ionizing radiation, is important for diagnosing ovarian tumors [8]. Straughn et al reported a patient with clinical abdominal tumors who had undergone abdominal CT scan and found solid tumors mixed with right adnexa cyst accompanied by increased serum CA-125 level. The patient underwent total abdominal hysterectomy and bilateral salpingoophorectomy and omentectomy, where anatomical pathology examination was performed. The results came out as tuberculosis, and the patient was given anti-tuberculosis drugs and showed improvement after therapy [9].

It is very common to find discrepancy between abdominal tumor's preoperative diagnosis, where its CT scan findings suggest ovarian tumor accompanied by increased CA-125 level, and its postoperative diagnosis, where it turns out to be an abdominal tuberculosis. Therefore, this study aims to determine the correlation between variations in serum CA-125 levels with variations in abdominal CT scan findings in patients with abdominal TB resembling ovarian mass and bacteriological and/or histopathological examinations results are available.

\section{Materials and Methods}

\subsection{Study Design}

This was a correlative analytic study with a retrospective approach which was conducted at Dr. Soetomo General Hospital, Surabaya, from January 2015-December 2020. This study was approved by the local Research Ethics Committee.

\subsection{Study Population and Sample}

The study population was all patients with clinical abdominal tumors who were tested for serum CA-125 level and underwent abdominal CT scan with contrast, and performed laparotomy followed by biopsy or tumorectomy, and histopathological and/or bacteriological examinations were performed to detect tuberculosis infection. The sample of this study was medical records from all patients with abdominal tuberculosis who had undergone histopathological examination from January 2015-December 2020 and met the inclusion criteria and was not included in the exclusion criteria.

The inclusion criteria were as followed:

- Patients with clinical abdominal tumor

- Serum CA-125 level has been examined

- Contrast-enhanced abdominal CT scan had been performed

- Laparotomy had been performed following a biopsy or tumorectomy

- Bacteriology or histopathology had been performed to detect tuberculosis infection

The exclusion criteria in this study were as followed:

- CT scan images was not feasible for evaluation

- Incomplete and unclear medical records

2.3. Data Collection

Serum CA-125 levels, surgery, and histopathological examination data were obtained from medical records. Data on serum CA-125 levels were sorted from lowest to highest value, then divided into 3 groups based on the classification from a previous study, which were:

- Low (CA $125<35 \mathrm{u} / \mathrm{mL})$

- Medium (CA $12535-65 \mathrm{u} / \mathrm{mL})$

- High (CA 125> $65 \mathrm{u} / \mathrm{mL})$ [10].

Abdominal CT scan data were obtained in DICOM format and then re-evaluated by observers, which were the authors and radiologists specializing in breast and women's reproductive organs. Abdominal CT scan findings were recorded and classified based on the modified ovarian tuberculosis scoring:

- Mild degree, if the score was between 5-8 
- Moderate degree, if the score was between 9-12

- Severe degree, if the score $>12$

The observer recorded the evaluation results on work sheets. Afterwards, a statistical analysis was performed.

\subsection{Data Analysis}

Study data processing and analysis were conducted through computerization using SPSS ver. 21 program. Correlation analysis between 2 groups of ordinal data was performed.

\section{Results}

\subsection{Abdominal CT Scan Findings}

In this study, 22 samples met the inclusion criteria and did not met the exclusion criteria. The data obtained were grouped based on distribution of lesion extension on CT scan, including omental changes, mesenterial changes, peritoneal thickening, lymph node enlargement, ascites, ovarian capsule changes, and contrast enhancement. The characteristics of the abdominal tuberculous lesion extension are shown in Table 1.

Table 1. Characteristics of extension of abdominal tuberculosis lesion resembling ovarian mass

\begin{tabular}{lrr}
\hline \multicolumn{1}{c}{ Lesion Characteristic } & Present & Absent \\
\hline Omental changes & 17 & 5 \\
Mesenterial changes & 18 & 4 \\
Peritoneal thickening & 20 & 2 \\
Lymph node enlargement & 10 & 12 \\
Ascites & 21 & 1 \\
Ovarian capsule changes & 17 (nodular/lobular edge) & 5 (smooth edge) \\
Contrast enhancement & 13 (strong enhancement) & 9 (weak enhancement) \\
\hline
\end{tabular}

Table 2 outlines omental changes found in abdominal tuberculosis patients resembling ovarian tumors. Most of omental changes had a cake-like shape, which was found in 10 patients $(45.5 \%)$, followed by nodular type in 7 patients $(31.8 \%)$, and no omental changes was found in 5 patients $(22.7 \%)$.

Table 2. Distribution of omental changes

\begin{tabular}{lrr}
\hline \multicolumn{1}{c}{ Omental Changes } & Frequency (n) & \multicolumn{2}{c}{ Percentage } \\
\hline Cake-Like & 10 & $45.5 \%$ \\
Nodular & 7 & $31.8 \%$ \\
Absent & 5 & $22.7 \%$ \\
\hline Total & 22 & $100.0 \%$ \\
\hline
\end{tabular}

Table 3 outlines mesenterial changes in abdominal tuberculosis patients resembling ovarian tumor. From this study, we could find several forms of mesenterial changes, which were loss of mesenterial configuration in 5 patients $(22.7 \%)$, followed by nodular infiltration/mesentery thickening in 13 patients $(59.1 \%)$, and no mesenterial changes found in 4 patients $(18.2 \%)$. 
Table 3. Distribution of mesenterial changes

\begin{tabular}{lrr}
\hline \multicolumn{1}{c}{ Mesenterial Changes } & Frequency (n) & Percentage (\%) \\
\hline Loss of mesenterial configuration & 5 & $22.7 \%$ \\
Nodular infiltration / mesenterial & 13 & $59.1 \%$ \\
thickening & & \\
Absent & 4 & $18.2 \%$ \\
\hline Total & 22 & $100.0 \%$ \\
\hline
\end{tabular}

Table 4 outlines some of peritoneal thickening types that were found. Several types of peritoneal thickening were found, which were thickening with smooth edges in 12 patients (54.5\%), thickening with lobulated edges in 8 patients $(36.4 \%)$, and no thickening was found in 2 patients $(9.1 \%)$.

Table 4. Distribution of peritoneal thickening

\begin{tabular}{lrr}
\hline \multicolumn{1}{c}{ Peritoneal Thickening } & Frequency (n) & \multicolumn{2}{c}{ Percentage } \\
\hline Smooth edge & 12 & $54.5 \%$ \\
Lobulated edge & 8 & $36.4 \%$ \\
Absent & 2 & $9.1 \%$ \\
\hline Total & 22 & $100.0 \%$ \\
\hline
\end{tabular}

Lymph node enlargement was also assessed in this study. In Table 5, it was found that lymph node enlargement of less than $1 \mathrm{~cm}$ was found in 12 samples $(54.5 \%)$, while in 10 patients (45.5\%) lymph node enlargement reached more than $1 \mathrm{~cm}$.

Table 5. Distribution of lymph node enlargement

\begin{tabular}{|c|c|c|}
\hline Lymph Node Enlargement & Frequency (n) & Percentage $(\%)$ \\
\hline$<1 \mathrm{~cm}$ & 12 & $45.5 \%$ \\
\hline$>1 \mathrm{~cm}$ & 10 & $31.8 \%$ \\
\hline Total & 22 & $100.0 \%$ \\
\hline
\end{tabular}

Table 6 outlines the presence of ascites in abdominal tuberculosis patient. Ascites was found in 21 patients $(95.5 \%)$, while only one patient $(4.5 \%)$ had no ascites.

Table 6. Presence of ascites

\begin{tabular}{lrrr}
\hline & Ascites & Frequency (n) & Percentage (\%) \\
\hline Present & 21 & $95.5 \%$ \\
Absent & 1 & $4.5 \%$ \\
\hline Total & 22 & $100.0 \%$ \\
\hline
\end{tabular}

Ovarian capsule changes are described in Table 7. These changes were divided into three criteria: ovarian capsule with smooth edges which was found in 5 patients $(22.7 \%)$, lobulated edges in 14 patients $(63.6 \%)$, and nodular edge in 3 patients $(13.6 \%)$.

Table 7. Distribution of ovarian capsule changes

\begin{tabular}{lrr}
\hline \multicolumn{1}{c}{ Ovarian Capsule Changes } & Frequency (n) & Percentage (\%) \\
\hline Smooth edge & 5 & $22.7 \%$ \\
Lobulated edge & 14 & $63.6 \%$ \\
Nodular edge & 3 & $13.6 \%$ \\
\hline Total & 22 & $100.0 \%$ \\
\hline
\end{tabular}


The final characteristic that we assessed was contrast enhancement in solid ovarian parenchyma. These enhancements were divided into two categories: more than $76 \mathrm{HU}$, which was found in 13 samples $(59.1 \%$, and 50-75 HU, which was found in 9 patients (40.9\%). Table 8 outlines these findings in this study.

Table 8. Distribution of contrast enhancement

\begin{tabular}{lrr}
\hline \multicolumn{1}{c}{$\begin{array}{c}\text { Contrast Enhancement in Solid } \\
\text { Ovarian Parenchyma }\end{array}$} & Frequency (n) & Percentage (\%) \\
\hline$>76 \mathrm{HU}$ & 13 & $59.1 \%$ \\
$50-75 \mathrm{HU}$ & 9 & $40.9 \%$ \\
\hline Total & 22 & $100.0 \%$ \\
\hline
\end{tabular}

Table 9 outlines the distribution of the above findings. These data were input into a scoring system, which was modified ovarian tuberculosis scoring. There were 8 patients $(35.4 \%)$ with mild degree, 13 patients $(59.1 \%)$ with moderate degree, and only one patient $(4.5 \%)$ with severe degree.

Table 9. Distribution of ovarian tuberculosis lesion extension degree

\begin{tabular}{lrr}
\hline \multicolumn{1}{c}{$\begin{array}{c}\text { Ovarian Tuberculosis Extension } \\
\text { Degree }\end{array}$} & Frequency (n) & Percentage (\%) \\
\hline Mild degree & 8 & $35.4 \%$ \\
Moderate degree & 13 & $59.1 \%$ \\
Severe degree & 1 & $4.5 \%$ \\
\hline Total & 22 & $100.0 \%$ \\
\hline
\end{tabular}

\subsection{Serum CA-125 Level}

Besides examining the lesion extension degree, we also examined serum CA-125 levels in abdominal tuberculosis patients. Table 10 outlines these findings and divided the patients based on their serum CA-125 level categories, which were normal CA-125 level in 3 patients (13.6\%), mild increase in 3 patients $(13.6 \%)$, and significant increase in 16 patients $(72.7 \%)$

Table 10. Distribution of serum CA-125 level category

\begin{tabular}{lrr}
\hline \multicolumn{1}{c}{ Serum CA-125 Level Category } & Frequency (n) & Percentage (\%) \\
\hline Normal & 3 & $13.6 \%$ \\
Mild increase & 3 & $13.6 \%$ \\
Significant increase & 16 & $72.7 \%$ \\
\hline Total & 22 & $100.0 \%$ \\
\hline
\end{tabular}

\subsection{Correlation Between Serum CA-125 Level and Lesion Extension Degree}

Pearson's correlation test was performed to determine the correlation between serum CA-125 level and lesion extension degree. Based on statistical results, it was found that there was a negative correlation $($ rho $=$ 0.096 ) which was not significant ( $p=0.671$ with $\alpha=0.05$ ) between serum CA 125 -levels and extension degree of abdominal tuberculosis lesion resembling ovarian tumors.

\section{Discussion}

The CT scan results of abdominal tuberculosis patients who resembled ovarian tumor were assessed using modified ovarian tuberculosis scoring. The lowest score was 5, which was included in mild degree and the 
highest score was 13, which was included in severe degree category. The distribution of the lesion extension degree varied, but most were included in moderate degree.

Serum CA-125 level in abdominal tuberculosis patients resembling ovarian tumor were varied, with the lowest level being $0.73 \mathrm{U} / \mathrm{dL}$, to the highest level being $604.33 \mathrm{U} / \mathrm{dL}$. Serum CA-125 levels were divided into 3 categories, which were normal, mild increase, and significant increase. Most patients belonged to the significant increase category $(72.7 \%)$ with, mean serum CA-125 level of $210.83 \mathrm{U} / \mathrm{dL}$. These findings were consistent with previous studies, which reported that there was an increase in serum CA 125 levels with abdominal tuberculosis patients. This was caused by mesothelial cells inflammation and ascites, which triggered mechanical stress in intra-abdominal mesothelial cells $[6,11,12]$.

\subsection{Correlation Between Serum CA-125 Level and Abdominal Tuberculosis Lesion Extension Degree}

It was found that CA-125 levels were normal in one patient (4.5\%) with mild lesion extension and in 2 patients $(9.0 \%)$ with moderate lesion extension. Mild increase of CA-125 levels were found in one patient (4.5\%) with mild lesion extension and 2 patients $(9.0 \%)$ with moderate lesion extension. Furthermore, significant increase of CA-125 levels were found in 6 patients $(27.3 \%)$ with mild lesion extension, 9 patients $(40.9 \%)$ with moderate lesion extension, and one patient $(4.5 \%)$ with severe lesion extension.

The above findings were then tested for their correlation with Pearson correlation test. It was found that there was negative correlation with no significance between serum CA-125 level and extension degree of abdominal tuberculosis lesion resembling ovarian tumor. These results might be due to small number of samples in this study and a more updated modified abdominal tuberculosis scoring needs to be developed.

\section{Conclusion}

There is a negative and insignificant correlation between serum CA-125 level and the degree of abdominal tuberculosis lesion extension resembling ovarian tumor. Further studies are needed with a larger population and a longer observation period. In addition, it is also necessary to conduct further studies on modified abdominal tuberculosis scoring.

\section{Acknowledgements}

I would like to dedicate my gratitude to all the lecturers of Radiology, Faculty of Medicine Airlangga University, Soetomo General Academic Hospital, my family, and my friends of their endless suppoert and prayers.

\section{References}

1. Kementerian Kesehatan RI (2018) 'InfoDatin Tuberculosis', Kementerian Kesehatan RI, p. 1. Available at: https://www.depkes.go.id/article/view/18030500005/waspadai-peningkatan-penyakit-

menular.html\%0Ahttp://www.depkes.go.id/article/view/17070700004/program-indonesia-sehat-dengan-pendekatan-keluarga.html.

2. Sah, S. K., Shi, X., Du, S., Li, X., Li, C. H., Shah, S., ... \& Li, Y. (2017). CT findings and analysis for misdiagnosis of female pelvic tuberculosis. Radiology of Infectious Diseases, 4(1), 19-25.

3. Rabesalama, S. S., Mandeville, K. L., Raherison, R. A., \& Rakoto-Ratsimba, H. N. (2011). Isolated ovarian tuberculosis mimicking ovarian carcinoma: case report and literature review. African journal of infectious diseases, 5(1).

4. Fendrick, J. L., Staley, K. A., Gee, M. K., McDougald, S. R., \& Quirk Jr, G. (1993). Characterization of CA 125 synthesized by the human epithelial amnion WISH cell line. Tumor biology, 14(5), 310-318.

5. Halila, H., Stenman, U. H., \& Seppälä, M. (1986). Ovarian cancer antigen CA 125 levels in pelvic inflammatory disease and pregnancy. Cancer, 57(7), 1327-1329.

6. Agarwala, A., Dhua, A., Shamim, S., \& Roy, P. P. (2015). Ovarian tubercular abscess mimicking ovarian carcinoma: A rare case report. The Journal of Association of Chest Physicians, 3(1), 14. 
7. Javadi, S., Ganeshan, D. M., Qayyum, A., Iyer, R. B., \& Bhosale, P. (2016). Ovarian cancer, the revised FIGO staging system, and the role of imaging. American Journal of Roentgenology, 206(6), 1351-1360.

8. Jeong, Y.-Y., Outwater, E. K. and Kang, H. K. (2000) 'Imaging Evaluation of Ovarian Masses', RadioGraphics, 20(5), pp. 1445-1470. doi: 10.1148/radiographics.20.5.g00se101445.

9. Straughn, J. M., Robertson, M. W. and Partridge, E. E. (2000) 'A patient presenting with a pelvic mass, elevated CA-125, and fever', Gynecologic Oncology, 77(3), pp. 471-472. doi: 10.1006/gyno.2000.5756.

10. Kimura, E., Murae, M., Koga, R., Odawara, Y., Nakabayashi, Y., Yokoyama, K., ... \& Yasuda, M. (1984). Clinical significance of new tumor marker CA 125 in gynecological cancer--particularly usefulness in diagnosis of ovarian cancer. Nihon Sanka Fujinka Gakkai Zasshi, 36(11), 2121-2128.

11. Thakur, V., Mukherjee, U. and Kumar, K. (2001) 'Elevated serum cancer antigen 125 levels in advanced abdominal tuberculosis', Medical Oncology, 18(4), pp. 289-291. doi: 10.1385/MO:18:4:289.

12. Huang, F. et al. (2012) 'New mechanism of elevated CA125 in heart failure: The mechanical stress and inflammatory stimuli initiate CA125 synthesis', Medical Hypotheses, 79(3), pp. 381-383. doi: 10.1016/j.mehy.2012.05.042. 